\title{
Minimum MSE-Based MIMO-OFDM Precoded Spatial Multiplexing Systems with Limited Feedback
}

\author{
Feng She, Wen Chen, Hanwen Luo and Xiaoting Yang \\ Department of Electronic Engineering \\ Shanghai Jiaotong University, Shanghai , P. R. China, 200240 \\ Email: \{shefeng630;wenchen\}@sjtu.edu.cn
}

\begin{abstract}
This paper deals with design and performance analysis of transmit precoder optimization for MIMO-OFDM systems with limited feedback of channel state information (CSI). We assume that the receiver has perfect channel knowledge while the transmitter has only partial channel knowledge from limited feedback. We propose MSE-based optimal codebook design algorithm for MIMO-OFDM precoded spatial multiplexing systems under a specific average power constraint. The optimal precoder has the structure of a precoding and power allocation for each mode obtained by water-filling process. We derived the closed form solution for power allocation in the MSEsense. Simulation results show that the MSE-based codebook construction algorithm with hybrid design of power allocation and precoding can achieve better performance than that of equal power allocattion based codebook in the previous works.
\end{abstract}

\section{INTRODUCTION}

Multiple-input multiple-output (MIMO) system is well motivated for wireless communication through fading channels because it has the potential to improve transmission rate or diversity gain. The performance of a multi-antenna system depends on the degree of channel state information (CSI) available at the transmitter and the receiver [1]. However, in practical wireless systems, the transmitter could not obtain perfect CSI because of various factors, e.g., estimation error, feedback delay, feedback error, etc. For multi-antenna systems, even partial channel knowledge can be valuable in enhance system performance [2]. Thus, exploiting channel partial CSI at the transmitter in a MIMO wireless systems has attracted great attention recently [3], [4].

Transmit CSI can enhance MIMO system performance by using precoder. CSI at transmitter can be obtained by feedback from receiver in frequency division duplexing systems or by measuring the reverse channel in time division duplexing systems. Generally, it is assumed that perfect CSI is available at the receiver while the transmitter only get partial CSI or imperfect CSI. There are two ways to exploit partial CSI [4], [7]. One is to use the statistical characteristic of CSI [2], [7]; The other is to use limited feedback bits which indexing CSI. The latter way is to first quantize the channel at the receiver, and then send back the limited bits indexing the quantized CSI which is called codebook. One can design off-line a codebook containing transmission candidates [7], [8]. The previous works partial CSI based transmit precoding are based on various criteria such as the average signal to noise ratio (SNR) [9], [10], the outage probability [11], [12], and the symbol error rate [13], [14] etc. Optimal transmit algorithms for maximal capacity based limited feedback CSI have been investigated in [15]. However, those are all based on equally power allocation. On the other hand, the transmitter structure in the previous work do not coincide with the optimal transmitter structure in [16]- [20].

By dividing frequency-selective channels into an equivalent set of frequency-flat subchannels, orthogonal frequency division multiplexing (OFDM) has emerged as an attractive modulation scheme to handle frequency selective fading resulting from delay spreading by expanding the symbol duration [5], [6]. It is also an effective technique to combat inter-symbol interference (ISI) caused by wireless multi-path fading channel [6]. Our focus in this paper is to take the critical work of determining which CSI and by which way to be sent to the transmitter for MIMO-OFDM systems.

In this paper, we consider power allocation across different subcarriers. We propose hybrid precoding and power adaptive allocation algorithm based on limited feedback with spatial multiplexing. Meanwhile, the minimal MSE based codebook design algorithm is also investigated.

The paper is organized as follows. We outline MIMOOFDM model and the problem statement in section II. In section III, the MSE design criterion is described. The minimum MSE based coodbook construction algorithm is presented in section IV. Section V proposes the optimal precoder design algorithm for MIMO-OFDM system with limited feedback. The simulation result are presented in section VI followed by the conclusion in section VII.

\section{SySTEM MODEL}

We consider $N_{t}$ transmitter-antennas and $N_{r}$ receiver antennas MIMO-OFDM wireless communication system with spatial multiplexing, where $N_{c}$ subcarriers is employed in an OFDM symbol. By MIMO-OFDM system, frequencyselective channel can be decoupled into $N_{c}$ parallel MIMO frequency-flat channels.

Assume that there are $N_{s}$ symbols to be transmitted per subcarrier, which is then bounded by $\min \left(N_{t}, N_{r}\right)$. Collect the $N_{c} N_{s}$ symbols transmitted over $N_{c} N_{t}$ subcarriers in a 


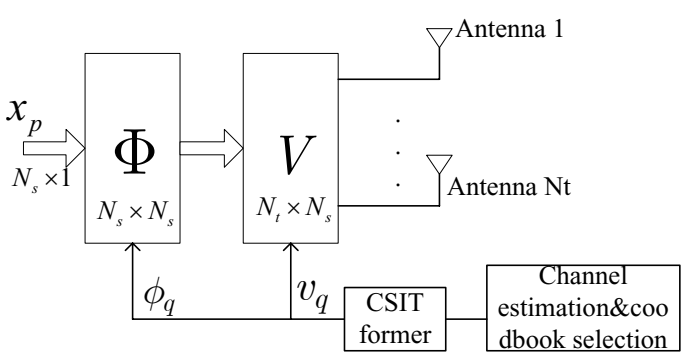

Fig. 1. The optimal transmission structure on each subcarrier

$N_{c} N_{s} \times 1$ vector $x$. The precoder is applied to each subcarrier to allocate power across the $N_{c}$ subcarriers and $N_{t}$ antennas. Therefore the precoder $\boldsymbol{F}$ is an $N_{c} N_{t} \times N_{c} N_{s}$ block diagonal matrix with $N_{c}$ blocks of $N_{t} \times N_{s}$ matrices $\boldsymbol{F}_{p}, p=1, \cdots, N_{c}$.

In this context, we consider a MIMO block-fading channel model where the channel state remains quasi-statistic within a fading block on each subcarrier, but behaves independently across a different fading block. The frequency responses of the $N_{c}$ MIMO channels can be described by a $N_{c} N_{r} \times N_{c} N_{t}$ block diagonal channel matrix. $\boldsymbol{H}$ with $N_{c}$ blocks of $N_{r} \times N_{t}$ matrices $\boldsymbol{H}_{p}, p=1, \cdots, N_{c}$. If the channel keeps quasi-static, the $N_{c} N_{s} \times 1$ received symbol vector $\mathbf{r}$ is

$$
\boldsymbol{r}=\boldsymbol{G H F x}+\boldsymbol{G n}
$$

where the receiver $\boldsymbol{G}$ is an $N_{c} N_{s} \times N_{c} N_{r}$ block diagonal matrix with $N_{c}$ blocks of $N_{s} \times N_{r}$ matrices $\boldsymbol{G}_{p}, p=1, \cdots, N_{c}$, and $\boldsymbol{n}$ is the $N_{c} N_{s} \times 1$ noise vector, which has the Gaussian statistics.

In a MIMO-OFDM system, each subcarrier can be considered as a MIMO channel. Suppose $\boldsymbol{x}^{T}=\left[\begin{array}{lll}\boldsymbol{x}_{1}^{T} & \cdots & \boldsymbol{x}_{N_{c}}^{T}\end{array}\right]$, $\boldsymbol{r}^{T}=\left[\begin{array}{lll}\boldsymbol{r}_{1}^{T} & \cdots & \boldsymbol{r}_{N_{c}}^{T}\end{array}\right]$ and $\boldsymbol{n}^{T}=\left[\begin{array}{lll}\boldsymbol{n}_{1}^{T} & \cdots & \boldsymbol{n}_{N_{c}}^{T}\end{array}\right]$, where $\boldsymbol{x}_{k}, \boldsymbol{r}_{k}$ and $\boldsymbol{n}_{k}$ are respectively the transmitted symbol vector, the received symbol vector and the noise vector over the $k$ th MIMO channel for $k=1, \cdots, N_{c}$. From (1), the output-input relation for each subcarrier can be individually written out. For $p=1, \ldots, N_{c}$, denote

$$
\boldsymbol{y}_{p}=\boldsymbol{H}_{p} \boldsymbol{F}_{p} \boldsymbol{x}_{p}+\boldsymbol{n}_{p} .
$$

Then for $p=1, \cdots, N_{c}$, we have

$$
\boldsymbol{r}_{p}=\boldsymbol{G}_{p} \boldsymbol{y}_{p}=\boldsymbol{G}_{p} \boldsymbol{H}_{p} \boldsymbol{F}_{p} \boldsymbol{x}_{p}+\boldsymbol{G}_{p} \boldsymbol{n}_{p}
$$

\section{Minimum MSE BAsed Codebook Construction}

\section{A. Transmitter Optimization}

Given an independent identical distribution (i.i.d.) blockfading MIMO channel with the transmit CSI of $B$ bit. The average minimum mean square error (MSE) $P_{e}(V, \Phi)$ of the MIMO-OFDM system can be computed from the $B$ bit index codeword. This optimal performance problem can be formulated as

$$
\left\{\begin{array}{l}
\min P_{e}(V, \Phi), \\
s . t . E\left(\sum_{p=1}^{N_{c}} \operatorname{tr}\left(\tilde{x}_{p} \tilde{x}_{p}^{H}\right)\right) \leq P_{0}
\end{array}\right.
$$

where $\tilde{\boldsymbol{x}}_{p}=V_{p} \sqrt{\Phi_{p}} \boldsymbol{x}_{p}$ and $P_{0}$ is the average transmit power.

\section{B. Optimal Transmitter Structure}

The proposed feedback transmission scheme in a MIMOOFDM system for one subcarrier is illustrated in Fig. 1. This is the optimal transmitter structure which hybrids adaptive power control and precoding model. Our objective is to jointly design the feedback scheme and the transmission parameters with limited feedback. Without loss of generality, in the following, we will not deliberately decern the $N_{c}$ MIMO systems and simply denote $\mathbf{H}_{p}, \Phi_{p}$ and $V_{p}$ for $p=1, \cdots N_{c}$ by $H, \phi$ and $v$ respectively.

For $B$ bits feedback, let $N_{B}=2^{B}$. There are $N_{B}$ candidates $v$ and $\phi$ in the codebook, denoted by $v(q)$ and $\phi(q)$ for $q=1, \cdots N_{B}$. The power loading matrice $\phi(q)$, and precoding matrices $v(q)$, for $q \in\left[1, N_{B}\right]$, are designed offline and stored at both transmitter and receiver. Each feedback index $q \in\left[1, N_{B}\right]$ is corresponding to a stored transmission scheme in the codebook. The power loading matrices are diagonal matrices with nonnegative entries. The column vectors of the precoding matrix are unitary. Without loss of generality, let $E\left[\boldsymbol{x}_{p}^{H} \boldsymbol{x}_{p}\right]=1$ for normalization.

Although the previous research has proposed many codebook construction criteria [9]-[11], there is no MSE based codebook construction criterion with optimal transmitter structure. [18] deduced the optimal MSE expression of linear transmitter and receiver, but it is based on perfect CSI on both sides. In this section we manage to directly use the exact MSE as the codebook design criterion. Let $\mathbf{W}$ be codebook designed off-line and known to the transmitter and the receiver, the proposed MSE based selection rule is

$$
\boldsymbol{F}_{o p t}=\arg \min _{\boldsymbol{F} \in \mathbf{W}} M S E(\boldsymbol{H}, \boldsymbol{F}) .
$$

The MSE expression can be computed by a simple expression in [20]. Let $P_{e}(\Phi)$ denote the MSE expression by allocated power $\Phi$, we can write it as

$$
P_{e}(\Phi)=\sum_{\ell=1}^{N_{s}} \frac{1}{1+\phi_{\ell} \lambda_{\ell}},
$$

where the constants $\lambda_{\ell}$ in (6) is singular value of channel SVD decomposition and $\phi_{\ell}$ is the power allocated on the $\ell$ th subchannel. In (6), we omitted the transmission scheme candidate index $q$.

\section{NMSE Based Codebook Construction Algorithm}

The codebook construction for limited-bit feedback beamforming and precoding can be linked to a vector quantization problem [8]. We use Lloyd algorithm to search for good precoder codebooks based on MSE criterion. The Lloyd algorithm based codebook construction also provides an alterative 
systematic approach for the subspace packing problem in [21], [22].

In Lloyd algorithm, the quantizer quantizes the input space $\mathbb{C}^{N}$ into $N_{B}$ separated regions $\left\{R_{q}\right\}_{q=1}^{N_{B}}$, where $R_{q}$ denote the region with codeword $\{v(q), \phi(q)\}$. Design a quantizer means to find a codebook and a partition rule that jointly minimize the overall average distortion measure [23], where the centroid condition and the nearest neighbor rule condition teratively play crucial roles.

To design the MSE based codebook, we have to construct "the nearest neighbor rule" and "the cenroid condition" in this scenario [4]. Thus, joint optimization of the channel vector region and the transmission modes is required. The two key works are

1) Given the channel vector region, find an optimal design of the transmission modes.

2) Given a set of transmission modes, find an optimal design of channel vector region.

Notice that the optimization of finding the regions $\left\{R_{1}, \cdots, R_{N_{B}}\right\}$, and transmission scheme $\{v, \phi\}_{q=1}^{N_{B}}$ is equivalent to designing a vector quantizer with a modified distortion measure.

Let $A_{q}$ denote the probability that the channel matrix $H$ lies in the region $R_{q}$, i.e, $P\left(H \in R_{q}\right)=A_{q}$. $P_{0}$ stands for the average transmit power. By jointly designing $\left\{R_{q}\right\}_{q=1}^{N_{B}}$ and transmitter strategy $\left\{v_{q}, \phi_{q}\right\}_{q=1}^{N_{B}}$, our ultimate goal is to

$$
\left\{\begin{array}{l}
\min J=\sum_{q=1}^{N_{B}}\left(A_{q} \cdot p_{e}(q)\right) \\
\text { s.t. } \sum_{q=1}^{N_{B}}\left(A_{q} \sum_{\ell=1}^{N_{s}}\left(\phi_{\ell}(q)\right)\right) \leq P_{0}
\end{array}\right.
$$

Using Lagrange multiplier $\mu$, the distortion measure for optimizing the MSE performance is given by

$$
\begin{aligned}
& D\left(H, v_{q}, \phi_{q}\right)=\sum_{q=1}^{N_{B}} A_{q} \frac{1}{N_{s}} \sum_{\ell=1}^{N_{s}} \frac{1}{1+\phi_{\ell}(q) \lambda_{\ell}(q)} \\
& \mu\left(\sum_{q=1}^{N_{B}} A_{q} \sum_{\ell=1}^{N_{s}} \phi_{\ell}(q)-P_{0}\right) .
\end{aligned}
$$

The distortion measure is a function of $H$ and $\phi_{q}$. The partition index $q$ is sent to the transmitter, and the transmitter selects the optimal schemes by the index $q$. Hence, the optimization problem could be solved by general Lloyd's algorithm, which can be outlined in the following two steps.

S1: Given a certain channel condition regions of $\left\{R_{1}, \cdots, R_{N_{B}}\right\}$, find the optimal transmission scheme $\left\{T_{1}, \cdots, T_{N_{B}}\right\}$, where the transmitter scheme $T_{q} \triangleq\left\{v_{q}, \phi_{q}\right\}$ denotes the multimode beamfoming matrix and power allocation along different mode. The optimal transmission scheme $T_{k}$, is given by the generalized region centroid condition.

$$
T_{q}=\underset{T}{\arg \min } E_{H \in R_{q}}\left[D\left(H, T_{q}\right)\right] A_{q} .
$$

S2: Given a transmission scheme $\left\{T_{q}\right\}$, find the optimal channel regions $R_{q}, 1 \leq q \leq N_{B}$. The optimal region is given by the nearest neighbor rule.

$$
R_{q}=\left\{H: D\left(H, T_{q}\right) \leq D\left(H, T_{j}\right), \forall j=1, \cdots, N_{B}\right\}
$$

By iteratively using centroid condition and the nearest neighbor rule, the overall distortion will decrease monotonically. We will use this principle to form a new codebook construction method. Here, we give the closed form solution for the power loading.

1) Solution of S1: For a given certain region $R_{q}, 1 \leq q \leq$ $N_{q}$, find the optimal transmission scheme $T_{q}$.

Given a certain region $R_{q}$, the probability $A_{q}$ and the channel covariance matrix can be calculated for every $q$ th region. Let the eigen decomposition of $H^{H} H$ in region $R_{q}$ be $H^{H} H=V_{q} \Lambda_{q} V_{q}^{H}$, where $\Lambda_{q}=\operatorname{diag}\left(\lambda_{1}(q), \cdots, \lambda_{N_{t}}(q)\right)$ is an $N_{t} \times N_{t}$ diagonal matrix with $\lambda_{1}(q) \geq \lambda_{2}(q) \geq \cdots \geq \lambda_{N_{t}}(q)$, and $V_{q}$ is $N_{t} \times N_{t}$ unitary matrix formed by corresponding eigen-vectors $v_{i}(q)$, i.e., $V_{q}=\left(v_{1}(q), \cdots, v_{N_{t}}(q)\right)$. The optimal unquantized precoder for each channel condition should be $\boldsymbol{F}_{\text {opt }}=\overline{\boldsymbol{V}}_{q}$, where $\overline{\boldsymbol{V}}_{q}$ is the matrix constructed from the first $N_{s}$ columns of $\boldsymbol{V}_{q}$ [4], [24]. Based on those decomposition matrices, we can derive the optimal power loading matrix belonging to the region $R_{q}$ consequently, which is summarized in the following theorem.

Theorem 1: With optimal eigen-beamforming combined with power allocation, we get the optimal transmitter power allocation schemes by water-filling principle:

$$
\phi_{\ell}(q)=\left[\frac{P_{t o t}+\sum_{q=1}^{N_{B}} \sum_{\ell=1}^{\bar{N}_{s}} \lambda_{\ell}^{-1}(q)}{\sum_{q=1}^{N_{B}} \sum_{\ell=1}^{N_{s}} \lambda_{\ell}^{-1 / 2}(q)} \lambda_{\ell}^{-1 / 2}(q)-\lambda_{\ell}^{-1}(q)\right]^{+}
$$

where $(x)^{+}=\max (0, x), q=1, \cdots, N_{B}$, and $\phi_{\ell}(q)$ denotes the $\ell$ th entry of the diagonal power allocation matrix of the qth region.

Since the worst eigen mode would be drop off in some cases, we have $\bar{N}_{s} \leq N_{s}$. Due to limited space, the proof is omitted. Notice that, the weaker subchannels may have a dominate MSE than the stronger subchannels. Thus, those eigenmodes whose channel gain are less than a certain threshold will be dropped, and the power is then distribute among the remaining eigenmodes. As a result, more power is allocated to the weaker eigenmodes to guarantee MSE performance.

2) Solution of $S 2$ : For a given transmission scheme, determine the optimal region $\left\{R_{q}\right\}_{q=1}^{N_{B}}$. The optimal partition region is given by the nearest neighbor rule. In our case, we will use the distortion measure as (8). Thus, we can get the new regions as following

$$
\begin{aligned}
R_{q}=\left\{H: D\left(H, T_{q}\right) \leq D\left(H, T_{j}\right)\right. & \\
& \left.\forall j \neq q \in\left[1, \cdots, N_{B}\right]\right\}
\end{aligned}
$$

The above two necessary optimization conditions are essential for the codebook construction [31]. Firstly, for each region, the optimal codeword can be chosen to minimize 
the distortion. In our case, precoding together with power allocation are employed in centroid condition. Secondly, the nearest neighbor rule is used to find the optimal region for each codeword.

3) Solution of Codewords Selection: Here, we present the optimal codeword selection algorithm for MIMO system. It can also be employed for MIMO-OFDM systems. By (2), our codeword selection algorithm can be employed on each sub-carrier. Let $T_{q}=\left\{w_{q}, \phi_{q}\right\}$ denote the $q$ th codeword in codebook $\left\{T_{q}\right\}_{q=1}^{N_{B}}$, then the description of the codewords selection is

St1 : Get CSI at receiver by channel estimation.

St2 : Perform eigen decomposition: $v^{H} H^{H} H v=V \Lambda V^{H}$, where $\Lambda=\operatorname{diag}\left(\lambda_{1}, \cdots, \lambda_{N_{s}}\right)$ and matrix $V$ denotes the eigen matrix of $v^{H} H^{H} H v$.

St3 : Let $D(H, T)=\sum_{\ell=1}^{N_{s}} \frac{1}{1+\lambda_{\ell} \phi_{\ell}}$, where $N_{s}$ denotes the data stream in each subcarrier. The selected optimal codeword is

$$
T^{o p t}=\arg \min _{T} D(H, T)
$$

\section{Simulation Result AND Discussion}

In this section, we provide some numerical examples to illustrate the performance of the optimal limited feedback design in section IV. We present Monte Carlo simulation for the MIMO-OFDM system with $N_{t}=4, N_{r}=2$. The number of sub-carriers is 64 , the cyclic prefix length is 16 and the used constellation is QPSK. We assume that the discretetime channel impulse response is generated according to the Hiperlan2 Channel Model C in [28]. The channels between different transmit and receiver antenna is assumed independent. The channel is fixed for a frame and randomly varies between frames. $n(k)$ is i.i.d complex Gaussian with zero mean. The transmitter power was allocated across subcarriers by waterfilling principle. The receiver uses linear decoder with perfect channel knowledge. Assume that the feedback CSI has no delay and no transmission error.

Experiment 1: The first experiment compares the performance of system with ideal CSI and partial CSI at the transmitter. When the transmitter has perfect CSI, best performance can be achieved. In partial CSI scenario, we compare the performance of MMSE based codebook and the existing codebooks in [7], [27]. We use actual Q-function in codebook selection criterion for the existing the codebook. Fig. 2 shows that MMSE-based codebook can get moderate performance gain over the existing codebook, and the codebook in [7] is a little bit better than that in [27]. We can also observe that Chernoff- bound approximation selection is not as good as actual Q-function selection.

Experiment 2: The codebook construction algorithm couples two steps: to find the optimal transmission $T_{1}, \cdots, T_{N_{B}}$ for a certain channel condition region and find the optimal channel regions $\left\{R_{k}\right\}$ for a transmission scheme $\left\{T_{k}\right\}$, for $k \in\left[1, N_{B}\right]$. The two steps works iteratively to enable the final design of the transmission modes and the fading regions. Fig. 3 shows that after three to four times of iterations, the distortion

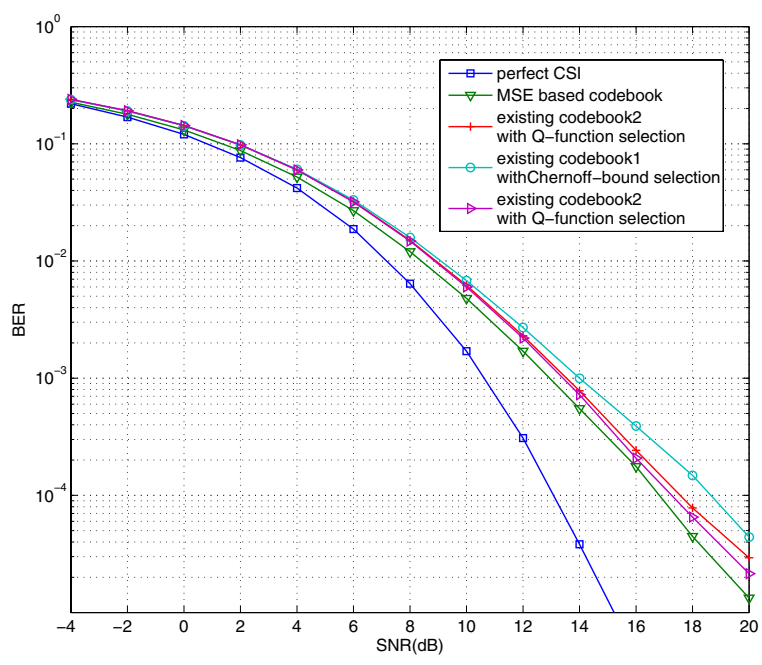

Fig. 2. BER performance comparison of optimal power allocation and codebook based limited feedback

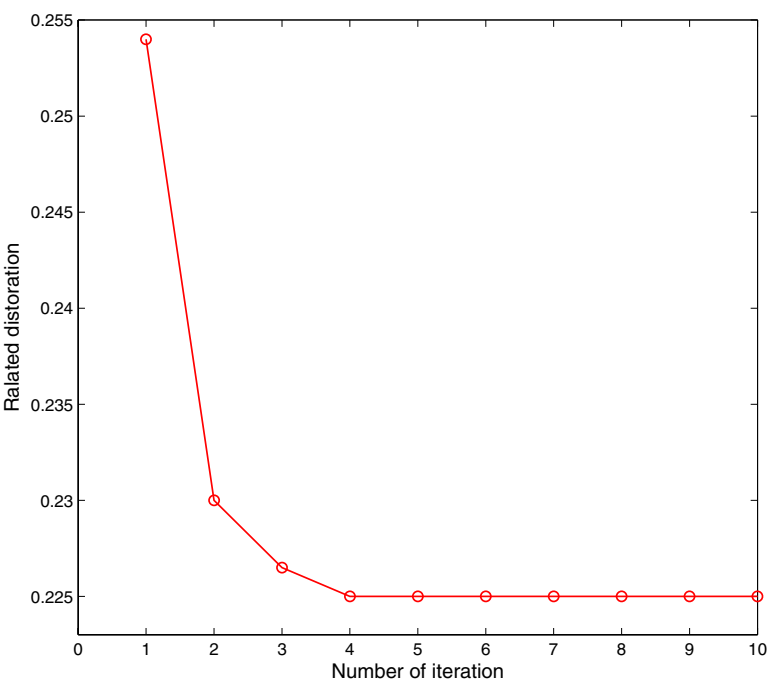

Fig. 3. Convergence of codebook construction algorithm

will converges. The Lloyd algorithm guarantees convergence in a few steps of iterations.

Experiment 3: The influence of size of the codebook is addressed in Fig. 4. The cases $B=4,6,7,8$ corresponds to the codebook sizes of $16,64,128,256$ respectively. Perfect CSI means $B=i n f$. From Fig. 4, we observe:

- Feedback link can improve the system performance;

- When the feedback bits increases, the system has performance gain with $B=\inf$ as the performance benchmark;

- the performance of $B=6$ is pretty good. The gap between $B=6, B=7$ and $B=8$ is small. So the size of codebook in a practical system is not necessarily 


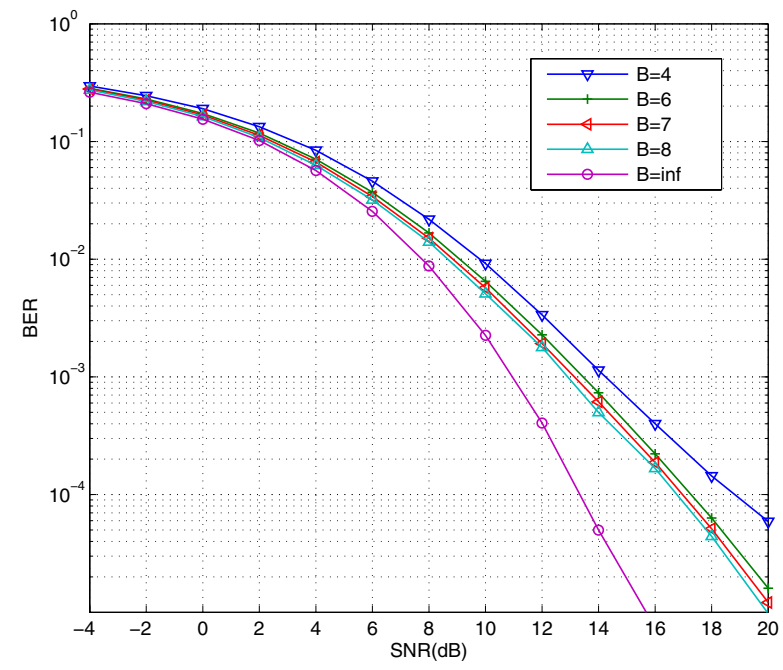

Fig. 4. The effect of codebook size

very large.

\section{CONCLUSION}

In this paper, we consider the precoded spatial multiplexing MIMO-OFDM system with limited feedback of CSI. We propose a new MMSE based codebook construction algorithm. The performance of the MMSE based codebook outperforms those of the existing codebooks. Then we develop the optimal algorithm for MIMO-OFDM system with limited feedback. The essential component of this paper is the codebook design using optimal structure of the transmitter and receiver, which employs the optimal transmission structure by combining precoding and power allocation. Since the codebook is designed offline, we do not need to care about the complexity of the presented codebook design algorithm.

\section{ACKNOWLEDGMENT}

This work is supported by NSF China \#60572157 and \#60672067, by NSF Shanghai \#062R14041, by ShanghaiCanada NRC \#06SN07112, by Cultivation Fund of the Key Scientific and Technical Innovation Project, Ministry of Education of China \#706022, and by Program for New Century Excellent Talents in University \#NCET-06-0386.

The authors would like to thank Xin Wang and Lei Chen for their helpful discussion.

\section{REFERENCES}

[1] S. A. Jafar, "Fundamental capacity limits of multiple antenna wireless systems," Ph.D. Dissertation, Stanford Univ., 2003.

[2] S. A. Jafar and A. Goldsmith, "Transmitter optimization and optimality of beamforming for multiple antenna systems," IEEE Trans. Wireless Commun., vol. 3, no. 4, pp. 1165-1175, July, 2004.

[3] F. Rey, M. Lamarca, "Robust power allocation algorithms for MIMO OFDM system with imperfect CSI," IEEE Trans. Signal Processing, vol. 53, no. 3, pp. 1070-1085, Mar., 2005.

[4] P. Xia, G. B. Giannakis, "Design and analysis of transmit-beamforming based on limited-rate feedback," IEEE Trans. Signal Processing, vol. 54, no. 5, pp. 1853-1863, May, 2006.
[5] V. Mignone and A. Morello, "CD3-OFDM: A novel demodulation scheme for fix and mobile receivers," IEEE Trans. Commun., vol. 44, no. 5, pp. 1144-1151, May, 1996.

[6] J. A. C. Bingham, "Multicarrier modulation for data transmission: an idea whose time has come," IEEE Trans. Commun., vol. 28, no. 5, pp. 1144-1151, May, 1990.

[7] S. L. Zhou, "BER criterion and codebook construction for finite-rate precoded spatial multiplexing with linear receiver," IEEE Trans. signal processing, vol. 54, no. 5, pp. 1653-1665, May, 2006.

[8] P. Xia, "Design and analysis of transmit-beamforming based on limitedrate feedback," IEEE Trans. Signal Processing. vol. 54, no. 5, pp. 18531863, May, 2006.

[9] D. J. Love and R. W. Heath Jr., "Diversity performance of precoded orthogonal Space-Time block codes using limited feedback," IEEE Commun. Lett, vol. 8, no. 5, pp. 305-307, May, 2004.

[10] D. J. Love and R. W. Heath Jr., "Limited feedback precoding for spatial multiplexing systems," in Proc. of Global Telecommunications Conference, pp. 1857-1861, San Francisco, CA, Dec., 2003.

[11] D. J. Love, R. W. Heath Jr., and T. Strohmer, "Grassmannian beamforming for multiple-input multiple-output wireless systems," IEEE Trans. Inform. Theory, vol. 49, no. 10, pp. 2735-2747, Oct., 2003.

[12] A. Narula, M. J. Lopez, M. D. Trott, and G. W. Wornell, "Efficient use of side information in multiple-antenna data transmission over fading channels," IEEE J. Select Areas Commun., vol. 16, no. 8, pp. 1423-1436, Oct., 1998.

[13] K. K. Mukkavilli, A. Sabharwal, E. Erkip, and B. Aazhang, "On Beamforming with Finite Rate Feedback in Multiple Antenna Systems," IEEE Trans. Inform. Theory, vol. 49, no. 10, pp. 2562- 2579, Oct., 2003.

[14] S. Zhou, Z. Wang, and G. B. Giannakis, "Quantifying the power-loss when transmit-beamforming relies on finite rate feedback," IEEE Trans. Wireless Commun., vol. 4, no. 4, pp. 1948-1957 Jul., 2005.

[15] V. Lau, Y. Liu, and T.-A. Chen, "On the design of MIMO block-fading channels with feedback-link capacity constraint," IEEE Trans. Commun., vol. 52, no. 1, pp. 62-70, Jan., 2004.

[16] M. Vu, A. Paulraj, "Optimal linear precoders for MIMO wireless correlated channels with nonzero mean in space-time coded systems," IEEE Trans. Signal Processing, vol. 54, no. 6, pp. 2318-2332, Jun., 2006.

[17] S. L. Zhou, "Optimal transmitter eigen-beamforming and space-time block coding based on channel mean feedback," IEEE Trans. Signal Processing, vol. 50, no. 10, pp. 2599-2613, Oct., 2002.

[18] A. Scaglione, P. Stoica, S. Barbarossa, "Optimal design for space-time linear precoders and decoders," IEEE Trans. Signal Processing, vol. 50, no. 5, pp. 1051-1064, May, 2002.

[19] D. P. Palomar, J. M. Cioffi, "Joint Tx-Rx beamforming design for multicarrier MIMO channels: a unified framework for convex optimization," IEEE Trans. Signal Processing, vol. 51, no. 9, pp. 2381-2401, Sep., 2003.

[20] H. Sampath, P. Stoica and A. Paulraj, "Generalized linear precoder and decoder design for MIMO channels using the weighted MMSE criterion," IEEE Trans. Commun., vol. 49, no. 12, pp. 2198-2206, Dec., 2001.

[21] J. H. Conway, R. H. Hardin, and N. J. A. Sloane, "Packing lines, planes, etc. Packings in Grassmannian spaces," Exper. Math., vol. 5, no. 2, pp. 139-159, 1996

[22] N. J. A. Sloane, "Packings in Grassmannian spaces," [Online] Available: http://www.research.att.com/ njas/grass/index.html.

[23] A. Gersho and R. M. Gray, "Vector Quantization and Signal Compression," Kluwer Academic Publishers, Boston, 1992.

[24] D. J. Love, R. W. Heath Jr., "Limited feedback unitary for spatial multiplexing system," IEEE Trans. Inform Theory, vol. 51, no. 8, pp. 2967-2976, Aug., 2005.

[25] D. J. Love, R. W. Heath Jr., "Limited feedback unitary precoding for orthogonal Space-Time block codes," IEEE Trans. signal processing, vol. 53, no. 1, pp. 64-73, Jan., 2005.

[26] P. Xia, "Multi-input multi-output communications with partial channel state information," Ph.D. Dissertation, Minesota Univ., 2004.

[27] D. Love, Personal Webpage on Grassmannian Subspace Packing, http://dynamo.ecn.purdue.edu/ djlove/grass.html.

[28] J. Medbo and P. Schramm, "Channel models for HIPERLAN/2 in different indoors scenarios," in ETSI/BRAN 3ERI085B, Mar. 1998. 
Authorized licensed use limited to: Shanghai Jiao Tong University. Downloaded on April 9, 2009 at 21:48 from IEEE Xplore. Restrictions apply. 